\title{
Design of a superpositively charged enzyme: human carbonic anhydrase II variant with ferritin
}

\section{encapsulation and immobilization}

Joshua A. Bulos, Rui Guo, Zhiheng Wang, Maegan A. DeLessio, Jeffery G. Saven, Ivan J. Dmochowski*

Department of Chemistry, University of Pennsylvania, Philadelphia, Pennsylvania, 19104

Fig. S1. Exterior residues, $\mathrm{K}+\mathrm{R}$ probability ranking. . S3

Fig. S2. Ramachandran plot of hCAII $(+21)$ residues S4

Fig. S3. SDS-PAGE analysis of overexpression of hCAII $(+23)$ S5

Fig. S4. SEC analysis of hCAII $(+23)$ mutant .S5

Fig. S5. SDS-PAGE analysis of hCAII $(+21)$ mutant S6

Fig. S6. CD thermal melts of hCAII wt and hCAII $(+21)$. S7

Fig. S7. Sequence alignment of hCAII wt and hCAII $(+21)$.... S7

Fig. S8. Acetazolamide inhibition of hCAII $(+21)$ S8

Fig. S9. SEC analysis of AfFtn-hCAII wt S8 


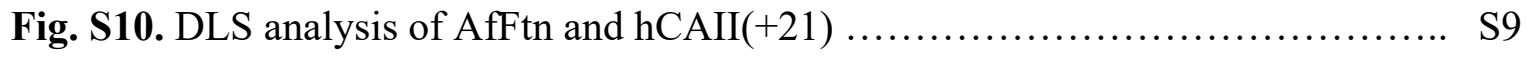

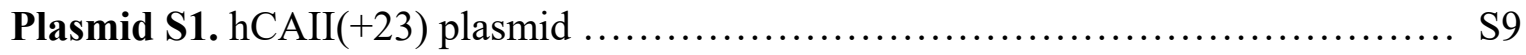




\section{Supporting Information}

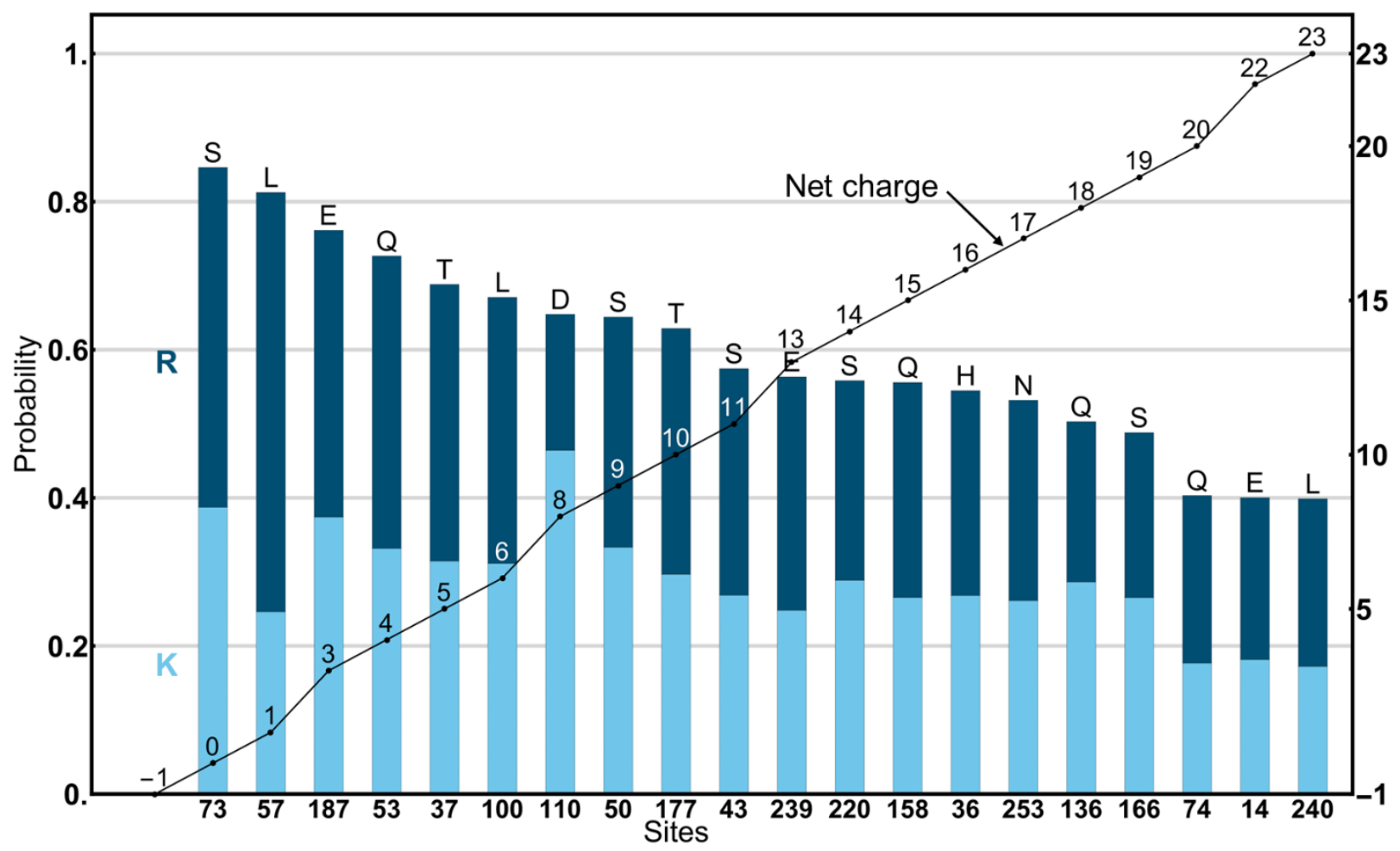

Figure S1. Probability $\left(\mathrm{p}_{+}\right)$of $\mathrm{K}$ and $\mathrm{R}$ for incorporation at exterior residues ranked from highest to lowest. This set has the largest values of $\mathrm{p}_{+}$among the 48 sites considered for mutation to increase positive charge. The probability of $\mathrm{K}$ is plotted as light blue bars, and the probability of $\mathrm{R}$ is plotted as dark blue bars stacking on top of $\mathrm{K}$ for each residue. The $\mathrm{x}$-axis indicates the site number, and the letter above the bars shows the wild type amino acid at the site. The line plot shows how the putative net charge at $\mathrm{pH} 7$ of hCAII changes if mutations (from wild type to $\mathrm{K}$ or R) are made in succession, where the starting charge of -1 at neutral $\mathrm{pH}$ is the net charge of wildtype hCAII. 


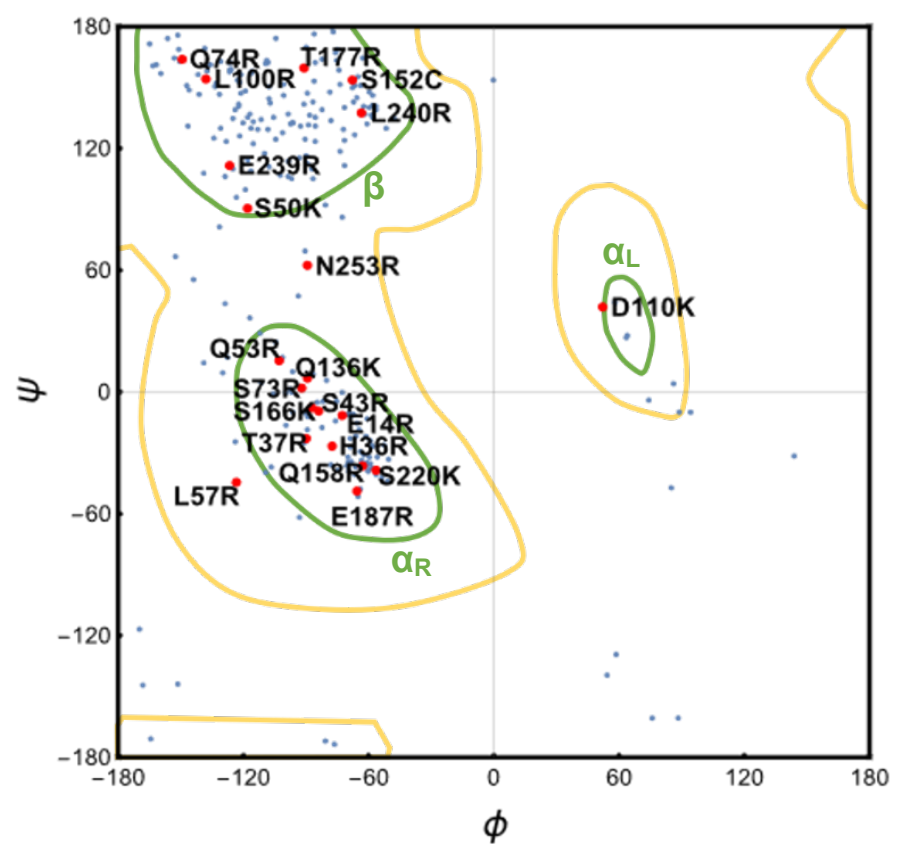

Figure S2. Ramachandran plot showing torsional angles, $\varphi$ and $\psi$, of all residues in hCAII. Designed 21 residues (including $\mathrm{S} 152 \mathrm{C}$ and 20 mutations) are indicated in red; remaining (nonmutated) residues are blue. Residue 110 is $\mathrm{D}(\mathrm{wt})$ in the +21 mutant hCAII $(+21)$. Green contours outline regions of secondary structure: $\beta$ sheet structures; $\alpha_{\mathrm{R}}$ right-handed $\alpha$-helices; and $\alpha_{\mathrm{L}}$ the $\alpha$-left region. 


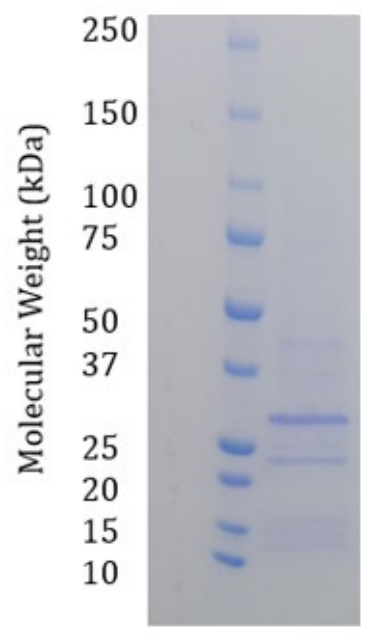

Figure S3. SDS-PAGE analysis of overexpression of hCAII(+23). SDS-PAGE of the insoluble fraction indicates that overexpression of the hCAII $(+23)$ mutant containing D110K resulted in inclusion bodies.

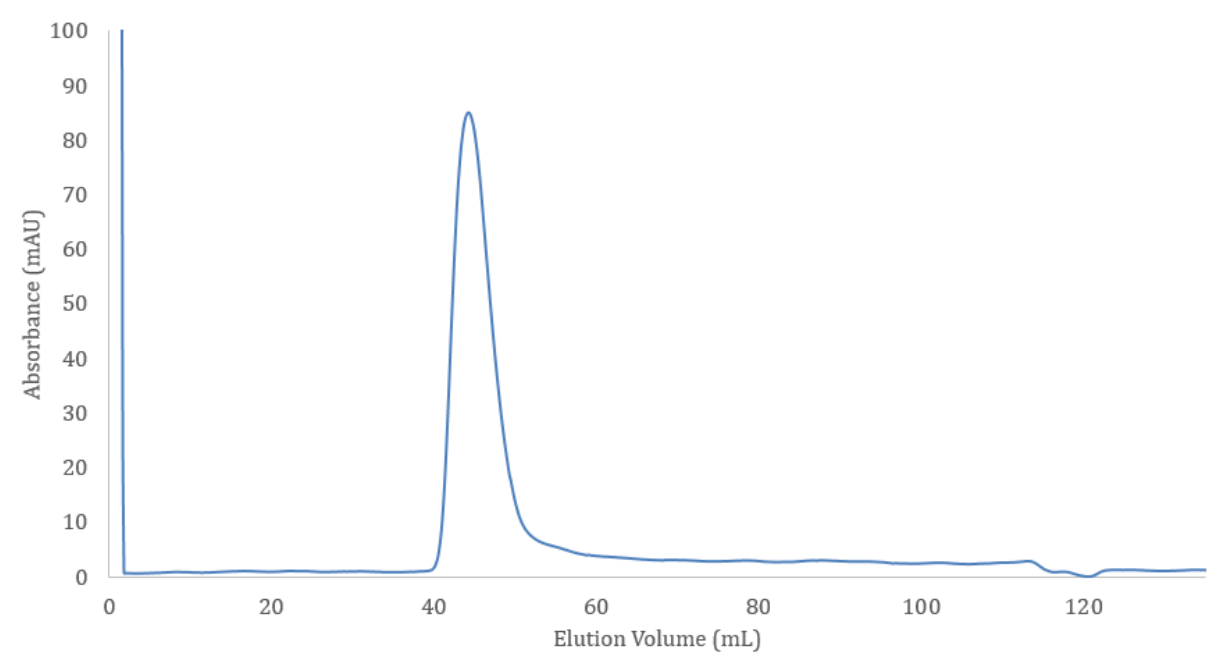

Figure S4. SEC analysis of hCAII $(+23)$ mutant. Elution of the small soluble fraction of D110Kcontaining mutant hCAII $(+23)$ results in inactive, soluble aggregates eluting in the void volume of the sizing column. 


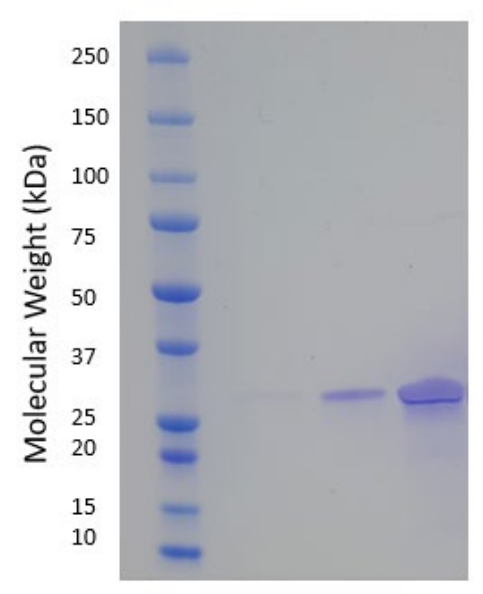

Figure S5. SDS-PAGE analysis of hCAII $(+21)$ mutant. SDS-PAGE of hCAII $(+21)$ indicates the correct MW and expected purity ( $>95 \%)$. 

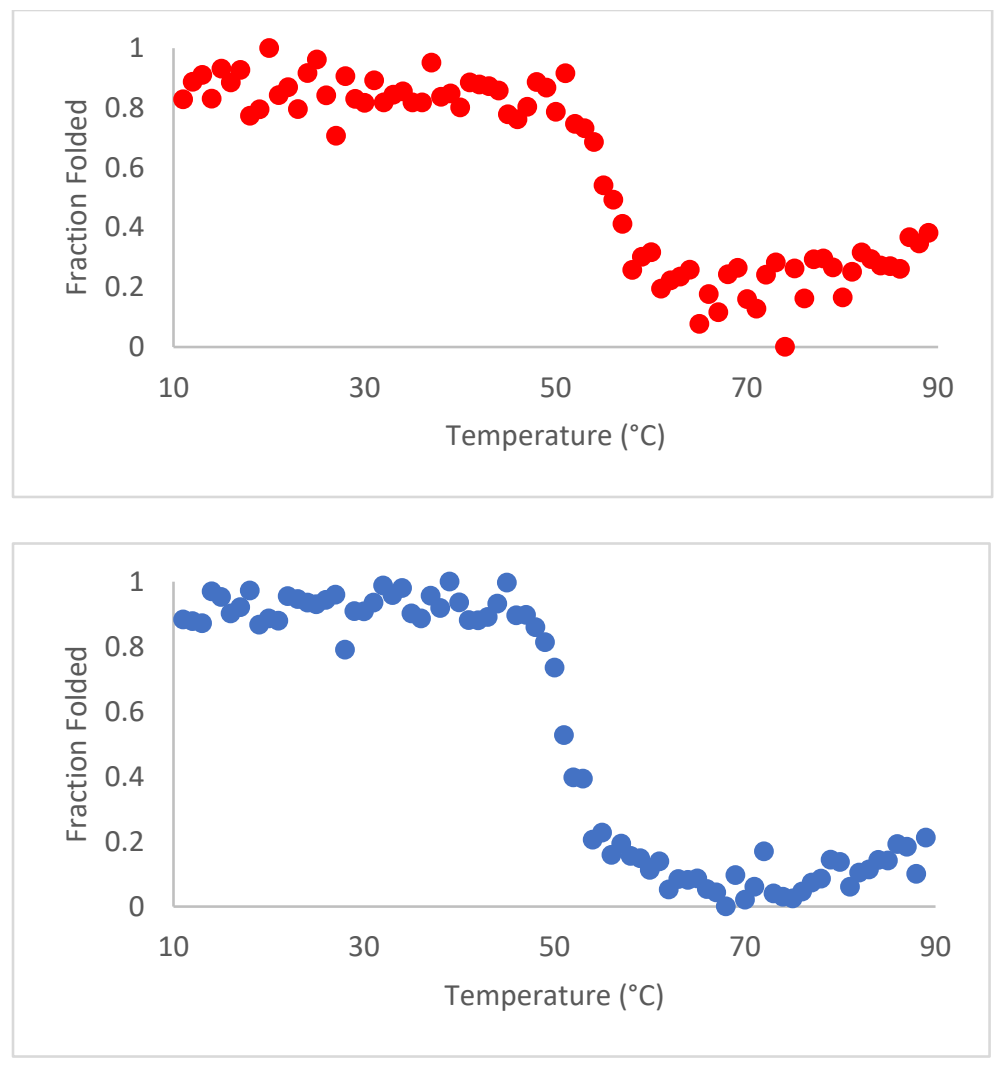

Figure S6. CD thermal melts of hCAII wt and hCAII $(+21)$ monitored at $205 \mathrm{~nm}$. CD thermal melting curves of hCAII wt (A) and hCAII $(+21)(\mathrm{B})$ show that the superpositively charged mutant loses only $4{ }^{\circ} \mathrm{C}$ of thermal stability relative to the wild-type enzyme $\left(\mathrm{Tm}=55^{\circ} \mathrm{C} \rightarrow 51^{\circ} \mathrm{C}\right)$.

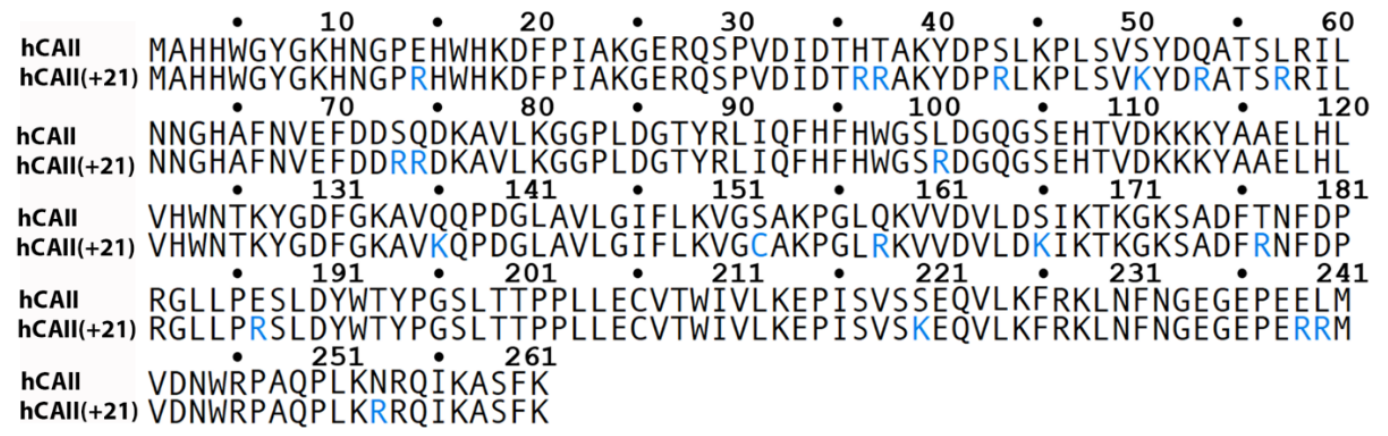

Figure S7. Sequence alignment of hCAII wt and hCAII $(+21)$. Changes between the wt and +21 sequence are shown in blue. 


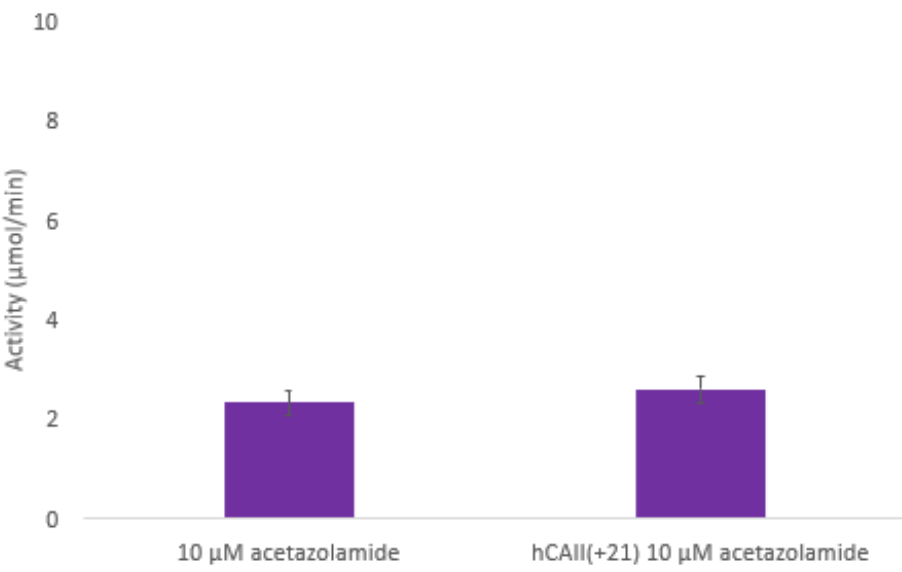

Figure S8. Inhibition of hCAII $(+21)$ by acetazolamide. hCAII $(+21)$ showed no pNPA esterase activity after adding $10 \mu \mathrm{M}$ acetazolamide, confirming that hCAII $(+21)$ is responsible for activity. Error bars indicate the standard error of triplicate measurements.



Figure S9. SEC analysis of AfFtn-hCAII wt at $0 \mathrm{mM} \mathrm{NaCl}$. AfFtn does not form 24mer cages around hCAII wt, as indicated by the elution of only a single peak corresponding to AfFtn dimer. 


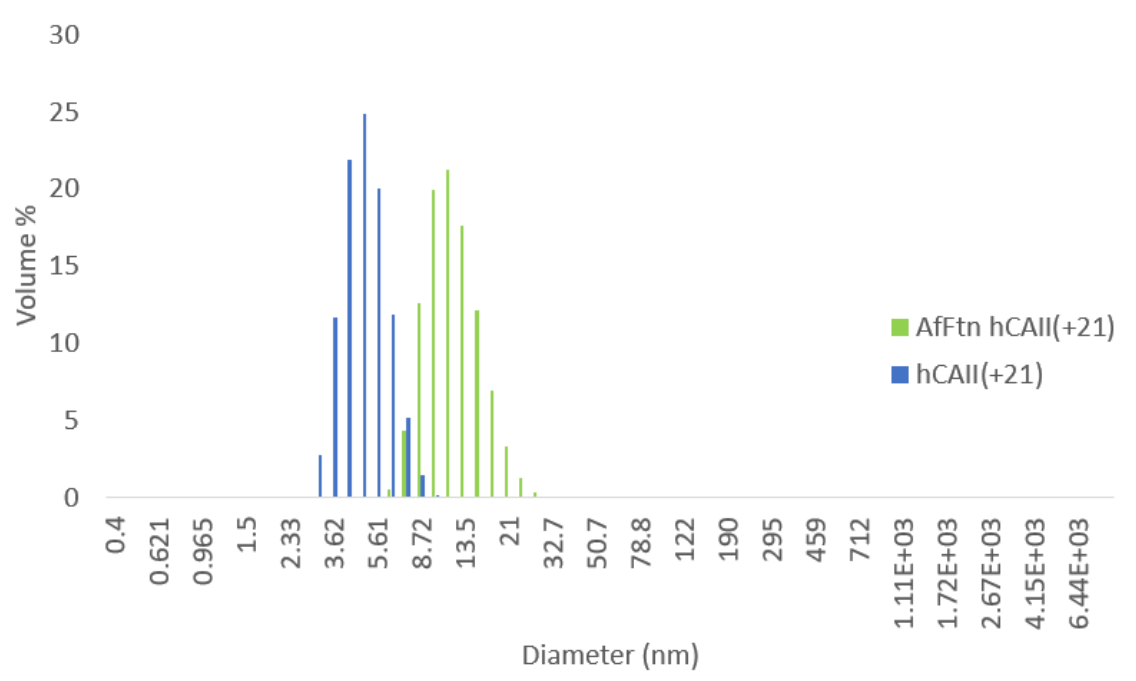

Figure S10. DLS analysis of hCAII $(+21)$ and AfFtn-hCAII $(+21)$. The purified host-guest complex shows size (roughly $13 \mathrm{~nm}$ diameter) that is consistent with AfFtn 24mer.

Plasmid S1. hCAII $(+23)$ plasmid

The plasmid for hCAII $(+23)$ was ordered from Atum bio using the vector pJ411 and the following insert:

AAGGAGGTAAAAAATGGCACATCACTGGGGTTACGGCAAGCACAACGGTCCACGT CACTGGCACAAAGATTTTCCGATCGCGAAGGGTGAGCGTCAGAGCCCGGTGGACAT TGACACCCGTCGCGCGAAATACGATCCGCGCCTGAAACCGCTGTCGGTCAAATATG ACCGTGCGACCTCCCGTCGTATTTTGAATAACGGCCATGCCTTCAACGTTGAGTTTG ACGACCGCCGTGATAAAGCCGTCCTGAAGGGTGGCCCGCTGGATGGTACGTACCGT CTGATTCAGTTTCACTTCCACTGGGGTAGCCGTGACGGTCAGGGCTCCGAGCATACC GTGAAAAAGAAAAAATACGCAGCGGAACTGCATCTGGTTCATTGGAACACGAAATA CGGTGATTTCGGTAAAGCTGTGAAGCAACCGGATGGTCTGGCTGTCCTGGGCATCTT TCTGAAAGTCGGCTGTGCGAAACCGGGCCTGCGCAAAGTTGTTGACGTGCTGGATA 
AGATCAAGACCAAGGGCAAGAGCGCGGACTTTCGTAACTTCGACCCTCGCGGTCTG TTGCCGAGAAGCCTGGACTATTGGACTTATCCTGGCAGCTTGACCACCCCGCCACTG CTGGAGTGCGTGACGTGGATCGTGCTGAAAGAACCGATCTCTGTTAGCAAAGAACA AGTCTTAAAGTTCCGCAAGCTGAATTTCAATGGTGAGGGTGAACCGGAGCGTCGCAT GGTTGATAATTGGCGTCCGGCACAACCGTTGAAGCGTCGCCAGATTAAGGCCAGCTT CAAGCACCACCATCATCACCACTAATGA 